\title{
Inoculation of a-synuclein preformed fibrils into the mouse gastrointestinal tract induces Lewy body-like aggregates in the brainstem via the vagus nerve
}

Norihito Uemura ${ }^{1 *}$ (D), Hisashi Yagi ${ }^{2}$, Maiko T. Uemura ${ }^{1}$, Yusuke Hatanaka ${ }^{1}$, Hodaka Yamakado ${ }^{1}$ and Ryosuke Takahashi ${ }^{*}$

\begin{abstract}
:
Background: Intraneuronal a-synuclein (a-Syn) aggregates known as Lewy bodies (LBs) and the loss of dopaminergic neurons in the substantia nigra pars compacta (SNpc) are the pathological hallmarks of Parkinson's disease (PD). Braak's hypothesis based on autopsy studies suggests that Lewy pathology initially occurs in the enteric nervous system (ENS) and then travels retrogradely to the dorsal motor nucleus of the vagus nerve (dmX), proceeding from there in a caudo-rostral direction. Recent evidence that a-Syn aggregates propagate between interconnected neurons supports this hypothesis. However, there is no direct evidence demonstrating this transmission from the ENS to the $\mathrm{dmX}$ and then to the SNpc.

Methods: We inoculated a-Syn preformed fibrils (PFFs) or phosphate-buffered saline (PBS) into the mouse gastric wall and analyzed the progression of the pathology.

Results: The mice inoculated with a-Syn PFFs, but not with PBS, developed phosphorylated a-Syn (p-a-Syn)positive LB-like aggregates in the $\mathrm{dmX}$ at 45 days postinoculation. This aggregate formation was completely abolished when vagotomy was performed prior to inoculation of a-Syn PFFs, suggesting that the aggregates in the $d m X$ were retrogradely induced via the vagus nerve. Unexpectedly, the number of neurons containing $p$-a-Synpositive aggregates in the $\mathrm{dmX}$ decreased over time, and no further caudo-rostral propagation beyond the $\mathrm{dmX}$ was observed up to 12 months postinoculation. P-a-Syn-positive aggregates were also present in the myenteric plexus at 12 months postinoculation. However, unlike in patients with PD, there was no cell-type specificity in neurons containing those aggregates in this model.

Conclusions: These results indicate that a-Syn PFF inoculation into the mouse gastrointestinal tract can induce a-Syn pathology resembling that of very early PD, but other factors are apparently required if further progression of PD pathology is to be replicated in this animal model.
\end{abstract}

Keywords: Parkinson's disease, a-synuclein, Lewy bodies, Propagation, Enteric nervous system, Vagus nerve

\footnotetext{
* Correspondence: nuemura@kuhp.kyoto-u.ac.jp; ryosuket@kuhp.kyoto-u.ac.jp ${ }^{1}$ Department of Neurology, Kyoto University Graduate School of Medicine, 54 Shogoin-Kawaharacho, Kyoto, Sakyoku 606-8507, Japan

Full list of author information is available at the end of the article
}

(c) The Author(s). 2018 Open Access This article is distributed under the terms of the Creative Commons Attribution 4.0 International License (http://creativecommons.org/licenses/by/4.0/), which permits unrestricted use, distribution, and reproduction in any medium, provided you give appropriate credit to the original author(s) and the source, provide a link to the Creative Commons license, and indicate if changes were made. The Creative Commons Public Domain Dedication waiver (http://creativecommons.org/publicdomain/zero/1.0/) applies to the data made available in this article, unless otherwise stated. 


\section{Background}

Parkinson's disease (PD) is the most common neurodegenerative movement disorder [1]. The pathologic findings include loss of dopaminergic neurons in the substantia nigra pars compacta ( $\mathrm{SNpc}$ ) and the presence of $\alpha$-synuclein ( $\alpha$-Syn) aggregates in the form of Lewy bodies (LBs) and Lewy neurites [2]. Clinically, PD is characterized by four cardinal motor manifestations: bradykinesia, muscle rigidity, resting tremor, and postural instability [3]. Other symptoms include various nonmotor features such as hyposmia, constipation, anxiety, depression, orthostatic hypotension, urinary dysfunction, rapid eye movement sleep behavior disorder, and cognitive dysfunction. Some of them are known as prodromal symptoms of PD or risk factors for developing the disease $[4,5]$.

Braak et al. systematically analyzed the pathology in cases with incidental LB pathology or sporadic PD and suggested that Lewy pathology initially develops in the olfactory bulb and the dorsal motor nucleus of the vagus nerve $(\mathrm{dmX})$ and then spreads in the brain stereotypically [6, 7]. Moreover, since Lewy pathology is found in the enteric nervous system (ENS) in the early stage of PD, they hypothesized that Lewy pathology in the ENS travels retrogradely to the $\mathrm{dmX}$ and then proceeds from there in a caudo-rostral direction [8]. Braak et al. integrated these observations into a staging system for PD consisting of six stages, each defined by Lewy pathology found in particular neuroanatomical structures. This staging system has gained much attention because it seems to explain the clinical course of PD well, from prodromal symptoms appearing early, to motor symptoms in the middle stage, and finally to cognitive dysfunction in the late stage [3].

Braak et al. proposed that an unknown neurotropic pathogen such as a virus initiates the pathogenesis underlying PD to explain the development of Lewy pathology in two independent sites: the olfactory bulb and the ENS [9]. In this scenario, the neurotropic pathogen is taken up by neurons and then progresses within nervous system by way of axonal transport and transsynaptic transmission. The observation that healthy neurons transplanted in the brains of patients with PD gradually developed LBs also suggested that certain pathogenetic events in PD are not cell-autonomous and raised the possibility that $\alpha$-Syn may be the propagating agent $[10,11]$. Afterwards animal studies have demonstrated that misfolded fibrillar forms of $\alpha$-Syn self-propagate and spread between interconnected neurons in the central nervous system (CNS), suggesting that cell-to-cell transmission of pathological proteins contributes to PD progression [12, 13].

Despite the great impact of the Braak's hypothesis on investigation of the clinicopathologic progression of PD, this hypothesis is still widely debated, with several issues needing resolution. For instance, it remains unclear whether Lewy pathology in the ENS indeed spreads to the $\mathrm{dmX}$, and, if so, how it travels on from the $\mathrm{dmX}$ to the SNpc [14, 15]. A previous study demonstrated that different forms of $\alpha$-Syn (i.e., PD brain lysate, monomeric, oligomeric, and fibrillar $\alpha$-Syn) inoculated into the rat intestinal wall traveled retrogradely in the vagus nerve and were found in $\mathrm{dmX}$ neurons at 6 days postinoculation [16]. However, it was not shown whether aggregated $\alpha$-Syn pathology formed in neurons to which inoculated $\alpha$-Syn has been transported [16]. Another study found that virus-mediated $\alpha$-Syn overexpression in the $\mathrm{dmX}$ and the ambiguous nucleus induced caudorostral progression of $\alpha$-Syn-positive neuritic pathology, but not somatic LB-like $\alpha$-Syn pathology [17].

Therefore, to address the issues described above, we inoculated $\alpha$-Syn preformed fibrils (PFFs) into the gastrointestinal tract of wild-type mice and observed the chronological progression of the pathology. We demonstrated that $\alpha$-Syn PFFs inoculated into the mouse gastric wall induced phosphorylated $\alpha$-Syn (p- $\alpha$-Syn)-positive LB-like aggregates in both the ENS and $d m X$. Further chronological analysis provides insights into the pathogenesis and progression of PD.

\section{Methods}

\section{Preparation of recombinant a-Syn monomers and preformed fibrils}

Mouse $\alpha$-Syn PFFs were generated as described previously with minor modifications [18]. Escherichia coli BL21 (DE3) (BioDynamics Laboratory) were transformed with plasmid pRK172 encoding the mouse $\alpha$-Syn cDNA sequence and incubated in LB medium to an optical density of 0.3 at $600 \mathrm{~nm}$. $\alpha$-Syn expression was induced by $0.1 \mathrm{mM}$ isopropyl $\beta$-D-1-thiogalactopyranoside for $4 \mathrm{~h}$. The bacteria were pelleted by centrifugation at $4000 \mathrm{~g}$ at $4{ }^{\circ} \mathrm{C}$ for $5 \mathrm{~min}$ and lysed by freeze/thaw and sonication. The lysate was clarified by boiling for $5 \mathrm{~min}$, followed by centrifugation at $20,400 \mathrm{~g}$ at $4{ }^{\circ} \mathrm{C}$ for $15 \mathrm{~min}$. The supernatant was subjected to ion exchange using Q sepharose fast flow (GE Healthcare), and $\alpha$-Syn was precipitated with 50\% (\% saturation) ammonium sulfate. Purified $\alpha$-Syn was dialyzed against dialysis buffer $(150 \mathrm{mM} \mathrm{KCl}, 50 \mathrm{mM}$ Tris- $\mathrm{HCl}, \mathrm{pH} 7.5)$ and cleared by ultracentrifugation at $186,000 \mathrm{~g}$ at $4{ }^{\circ} \mathrm{C}$ for $20 \mathrm{~min}$. The protein concentration was determined with a Pierce BCA Protein Assay kit (Thermo Fisher). Purified $\alpha$-Syn was diluted in dialysis buffer containing $0.1 \%(\mathrm{w} / \mathrm{v})$ $\mathrm{NaN}_{3}$ to $7 \mathrm{mg} / \mathrm{ml}$, followed by incubation at $37{ }^{\circ} \mathrm{C}$ in a shaking incubator (AS ONE, SI-300C) at $1000 \mathrm{rpm}$ for 10 days. The $\alpha$-Syn PFF pellet was obtained by ultracentrifugation at $186,000 \mathrm{~g}$ at $20{ }^{\circ} \mathrm{C}$ for $20 \mathrm{~min}$ and stored at $-80{ }^{\circ} \mathrm{C}$ until use. The pellet was dissolved in $8 \mathrm{M}$ 
guanidine hydrochloride to determine the protein concentration with a Pierce BCA Protein Assay kit (Thermo Fisher). The $\alpha$-Syn PFFs in phosphate-buffered saline (PBS) $(2 \mu \mathrm{g} / \mu \mathrm{l})$ were sonicated with an ultrasonic wave disruption system (Cosmo Bio, Bioruptor) for $2 \mathrm{~min}$ before inoculation into the mouse gastric wall.

\section{Sodium dodecyl sulfate-polyacrylamide gel electrophoresis and western blot analysis}

For sodium dodecyl sulfate (SDS)-polyacrylamide gel electrophoresis (PAGE), sample buffer (1\% [w/v] SDS, $12.5 \%$ [w/v] glycerol, 0.005\% [w/v] bromophenol blue, 2 . $5 \%$ [v/v] 2-mercaptoethanol, $25 \mathrm{mM}$ Tris- $\mathrm{HCl}, \mathrm{pH}$ 6.8) was added to $\alpha$-Syn monomer solution or the $\alpha$-Syn PFF pellet, which was resuspended by vortex. Samples containing $10 \mu \mathrm{g}$ protein were loaded in each lane and separated on $12 \%(w / v)$ gels for SDS-PAGE. For Coomassie Brilliant Blue $(\mathrm{CBB})$ staining, gels were incubated in CBB staining solution (0.1\% [w/v] PhastGel Blue R-350 [GE Healthcare], 30\% [v/v] methanol, 10\% [v/v] acetic acid) at room temperature. For western blot analysis, proteins were transferred to polyvinylidene difluoride membranes with a Trans-Blot SD Semi-Dry Transfer Cell (Bio-Rad). The membranes were treated with $0.4 \%$ $(\mathrm{w} / \mathrm{v})$ paraformaldehyde (PFA) in PBS for $30 \mathrm{~min}$ at room temperature before blocking with $5 \%$ skim milk to prevent detachment of $\alpha$-Syn from the blotted membranes [19]. The membranes were incubated with an anti- $\alpha$-Syn primary antibody (BD Transduction, \#610787 [Syn-1], 1:2000) at $4{ }^{\circ} \mathrm{C}$ overnight, followed by reaction with horseradish peroxidase-conjugated secondary antibodies (Santa Cruz, \#sc-2005, 1:5000) for $1 \mathrm{~h}$ at room temperature. Immunoreactive bands were detected with Pierce ECL Western Blotting Substrate (Thermo Fisher), and the chemiluminescent signal was detected with an Amersham Imager 600 imager (GE Healthcare).

\section{Thioflavin T assay}

$\alpha$-Syn monomers or PFFs, $7 \mu \mathrm{g}$ each, were incubated in $250 \mu \mathrm{l}$ of $5 \mu \mathrm{M}$ Thioflavin $\mathrm{T}$ (Sigma-Aldrich, \#T3516) solution for $15 \mathrm{~min}$ at room temperature. The fluorescence at $535 \mathrm{~nm}$ (excitation $450 \mathrm{~nm}$ ) was measured with a multi-label plate reader (PerkinElmer, 2030 ARVO X). Thioflavin T solution alone was measured as a blank.

\section{Transmission electron microscopy}

$\alpha$-Syn PFFs, $5 \mu \mathrm{g}$, were placed on a 400-mesh carboncoated copper grid (Nissin EM). The excess solution was removed with filter paper after the sample had stood for $1 \mathrm{~min}$. The PFFs adsorbed on the grid were negatively stained with a $2 \%(\mathrm{w} / \mathrm{v})$ uranyl acetate solution. Electron micrographs were acquired using a transmission electron microscope (JEOL, JEM-1400 Plus) at $80 \mathrm{kV}$.

\section{Animals and ethics statement}

C57BL/6J male mice at 2 months of age were used for the present study $(n=61)$. All experimental procedures used in this study followed national guidelines. The Animal Research Committee of Kyoto University granted ethical approval and permission (MedKyo 17,184).

\section{Inoculation of a-Syn PFFs into the mouse gastric wall}

Mice were anesthetized with Avertin (1.875\% [w/v] 2,2,2tribromoethanol, 1.25\% [v/v] 3-methyl-1-butanol). A 2-cm incision was made in the abdominal midline, followed by inoculation of $\alpha$-Syn PFFs $(n=48)$ or PBS $(n=3)$ into the gastric wall. Among the mice inoculated with $\alpha$-Syn PFFs, 8 mice underwent right cervical vagotomy just prior to inoculation of $\alpha$-Syn PFFs. Each of 8 sites was inoculated with $3 \mu \mathrm{l}$ of $\alpha$-Syn PFFs in PBS $(2 \mu \mathrm{g} / \mu \mathrm{l})$ or with PBS using a 37 gauge needle (Saito Medical Instruments) fitted to $10 \mu$ l syringe (Hamilton, \#701LT).

\section{Cervical vagotomy}

Following anesthesia, a $1-\mathrm{cm}$ incision was made at the midline of the mouse neck. The right vagus nerve was identified between the common carotid artery and the jugular vein behind the submandibular gland. After isolation from the carotid sheath, the vagus nerve was cut with a pair of tweezers.

\section{FluoroGold verification of vagotomy}

Verification of vagotomy using FluoroGold was performed as described with modifications [20]. Five days prior to sacrifice, unvagotomized or vagotomized mice (3 mice per group) were injected intraperitoneally with $1.2 \mathrm{mg}$ of hydroxystilbamidine (AAT Bioquest) in $1 \mathrm{ml}$ of saline. Frozen sections were obtained as described below and observed with a BZ-×710 fluorescence microscope (KEYENCE).

\section{Histologic and immunohistochemical analysis}

Frozen and paraffin sections were used for histologic and immunohistochemical analysis. Following perfusion with $4 \%(\mathrm{w} / \mathrm{v})$ PFA in PBS, the brains were removed and immersed in $4 \%(\mathrm{w} / \mathrm{v})$ PFA in PBS. For frozen sections, the brains were replaced in $15 \%(\mathrm{w} / \mathrm{v})$ sucrose in PBS and subsequently in $30 \%(\mathrm{w} / \mathrm{v})$ sucrose in PBS at $4{ }^{\circ} \mathrm{C}$ each overnight. The brains were embedded in Surgipath FSC 22 (Leica), and 10- $\mu$ m sections were obtained with a CM1950 cryostat (Leica). For paraffin sections, the mouse brains were dehydrated and embedded in paraffin, and $8-\mu \mathrm{m}$ paraffin sections were prepared with a HM 325 rotary microtome (MICROM). For immunohistochemical analysis, the following primary antibodies were used: anti- $\alpha$-Syn (BD Transduction, \#610787 [Syn-1], 1:1000), anti-p- $\alpha$-Syn (Abcam, \#ab51253 [EP1536Y], 1:10000), anti-p- $\alpha-S y n$ (Wako, 
\#015-25191 [\#64], 1:2000), anti-p- $\alpha-S y n$ (Abcam, \#ab184674 [81A], 1:5000), anti-nitrated $\alpha$-Syn (Santa Cruz, \#sc-32,279 [Syn514], 1:200), anti-choline acetyltransferase (ChAT) (Millipore, \#AB144P, 1:1000), antip62 (MBL, \#PM045, 1:1000), anti-ubiquitin (DAKO, \#Z0458, 1:500), anti-vasoactive intestinal polypeptide (VIP) (Abcam, \#ab8556, 1:50), anti-nitric oxide synthase 1 (NOS1) (Santa Cruz, \#A-11, 1:200), and antisubstance P (Millipore, \#MAB356, 1:200). The sections were incubated at $4{ }^{\circ} \mathrm{C}$ with primary antibodies for $2 \mathrm{~d}$ and then processed for visualization. As secondary antibodies, Histofine (Nichirei Bioscience) was used for diaminobenzidine staining, and Alexa Fluor 488 or 594conjugated antibodies (Molecular Probes) for immunofluorescence. For $\mathrm{p}-\alpha-$ Syn and Thioflavin S (ThS, Santa Cruz, \#sc-391005) double-labeling staining, after immunolabeled with $\mathrm{p}$ - $\alpha$-Syn antibody, slides were incubated with $0.05 \%$ ThS in $50 \%$ ethanol followed by differentiation with $80 \%$ ethanol. For assessment of p$\alpha$-Syn pathology, every $10^{\text {th }}$ paraffin section was stained with anti-p- $\alpha$-Syn antibody (EP1536Y). To assess ChAT-positive neurons in the $\mathrm{dmX}$, every $10^{\text {th }}$ paraffin section was stained with anti-ChAT antibody. The numbers of $\mathrm{p}-\alpha$-Syn-positive and ChAT-positive neurons were manually counted. Sections were examined with a BX43 microscope (Olympus), a BZ-X710 fluorescence microscope (KEYENCE), and an FV-1000 confocal laser scanning microscope (Olympus).

\section{Statistical analysis}

One-way ANOVA with Tukey's post-hoc test was used. Statistical calculations were performed with GraphPad Prism Software, Version 5.0.

\section{Results}

\section{Inoculation of a-Syn preformed fibrils into the mouse} gastric wall

We used mouse rather than human $\alpha$-Syn PFFs in this study because mouse $\alpha$-Syn PFFs are more potent for inducing $\alpha$-Syn pathology and its propagation as compared with human $\alpha$-Syn PFFs inoculated in wild-type mouse brains $[13,21]$. First, we generated mouse $\alpha$-Syn PFFs and validated their characteristics. SDS-PAGE followed by CBB staining or western blotting showed smear bands of $\alpha$-Syn PFFs, suggesting $\alpha$-Syn polymerization (Fig. 1a). High-intensity Thioflavin T fluorescence of $\alpha$-Syn PFFs indicated that $\alpha$-Syn PFFs comprised $\beta$-sheet-rich structures (Fig. 1b). Transmission electron microscopy revealed fibrillar structures in the $\alpha$-Syn PFFs (Fig. 1c). We inoculated $\alpha$-Syn PFFs or PBS into the gastric wall of the mice. To confirm that the PFFs properly inoculated into the gastric wall, we immunostained paraffin sections of the gastric wall with anti- $\alpha$-Syn antibody. Deposition of inoculated $\alpha$-Syn PFFs was found in the submucosal layer
(Fig. 1d, e) and in the muscular layer (Fig. 1g, h) even 45 days after $\alpha$-Syn PFFs inoculation. $\alpha$-Syn immunohistochemistry also showed $\alpha$-Syn-positive dots around the myenteric neurons, some of which are efferent vagus nerve terminals (Fig. 1f) [20].

\section{Phosphorylated a-Syn pathology was observed in the dmX 45 days after $a-S y n$ PFF inoculation}

Forty-five days after inoculation of $\alpha$-Syn PFFs into the gastric walls, we examined the brains and stomachs of the mice. Immunostaining for $\mathrm{p}-\alpha-\mathrm{Syn}$, a marker of human Lewy pathology, showed $\mathrm{p}-\alpha-\mathrm{Syn}-$ positive neurons in both rostral and caudal parts of the $\mathrm{dmX}$ in mice that had been inoculated with $\alpha$-Syn PFFs (Fig. 2a-f). All those neurons had $\mathrm{p}-\alpha-$ Syn in the cytoplasm, whereas a few also exhibited intranuclear $p$ - $\alpha$-Syn-positive dots (Fig. 2c). We confirmed that the pathology in the $\mathrm{dmX}$ were detectable with other anti-p- $\alpha$-Syn antibodies and an anti-nitrated $\alpha$-Syn antibody (Fig. 2g-i) [22]. However, p- $\alpha$-Syn pathology were rarely observed in the stomachs 45 days after $\alpha$-Syn PFF inoculation (Additional file 1: Figure S1), nor were they seen in either brains or stomachs of mice inoculated with only PBS (Additional file 2: Figure S2).

\section{Phosphorylated a-Syn pathology exhibited LB-like features} Next, we examined the pathological features of the $p-\alpha$ Syn pathology in the $\mathrm{dmX}$. We confirmed that it was present in ChAT-positive neurons of the dmX (Fig. 3a-d). The $\mathrm{p}-\alpha$-Syn pathology was positive for ThS, indicating that they were composed of $\beta$-sheet-rich amyloid fibrils (Fig. 3e-h). Most of the $\mathrm{p}-\alpha$-Syn pathology was also immunopositive for p62 (Fig. 3i-l) and ubiquitin (Fig. $3 \mathrm{~m}-\mathrm{p}$ ), both of which are LB markers. All these observations indicated that the $\mathrm{p}-\alpha$-Syn pathology in the $\mathrm{dmX}$ shared properties in common with the LBs seen in patients with PD.

\section{Vagus nerve mediated the formation of $p-a-S y n$ pathology in the $\mathrm{dmX}$}

Anatomically, it was presumed that $\alpha$-Syn PFFs inoculated into the gastric wall retrogradely induced $\mathrm{p}-\alpha-\mathrm{Syn}-$ positive LB-like aggregates in the $\mathrm{dmX}$ via the vagus nerve. To verify this, we inoculated $\alpha$-Syn PFFs into the gastric wall immediately after hemivagotomy. We confirmed that vagotomy was successful using a retrograde neuronal tracer, FluoroGold. Intraperitoneal injection of FluoroGold labeled neurons in the $\mathrm{dmX}$ as has been reported in rats (Fig. 4a) [20]. When FluoroGold was injected after hemivagotomy, neurons on the vagotomy side were not labeled, indicating that the vagotomy had succeeded (Fig. 4a). However, it was reported that vagotomy causes neuronal loss over time in the murine $\mathrm{dmX}$ on the vagotomy side [23], so we analyzed some 


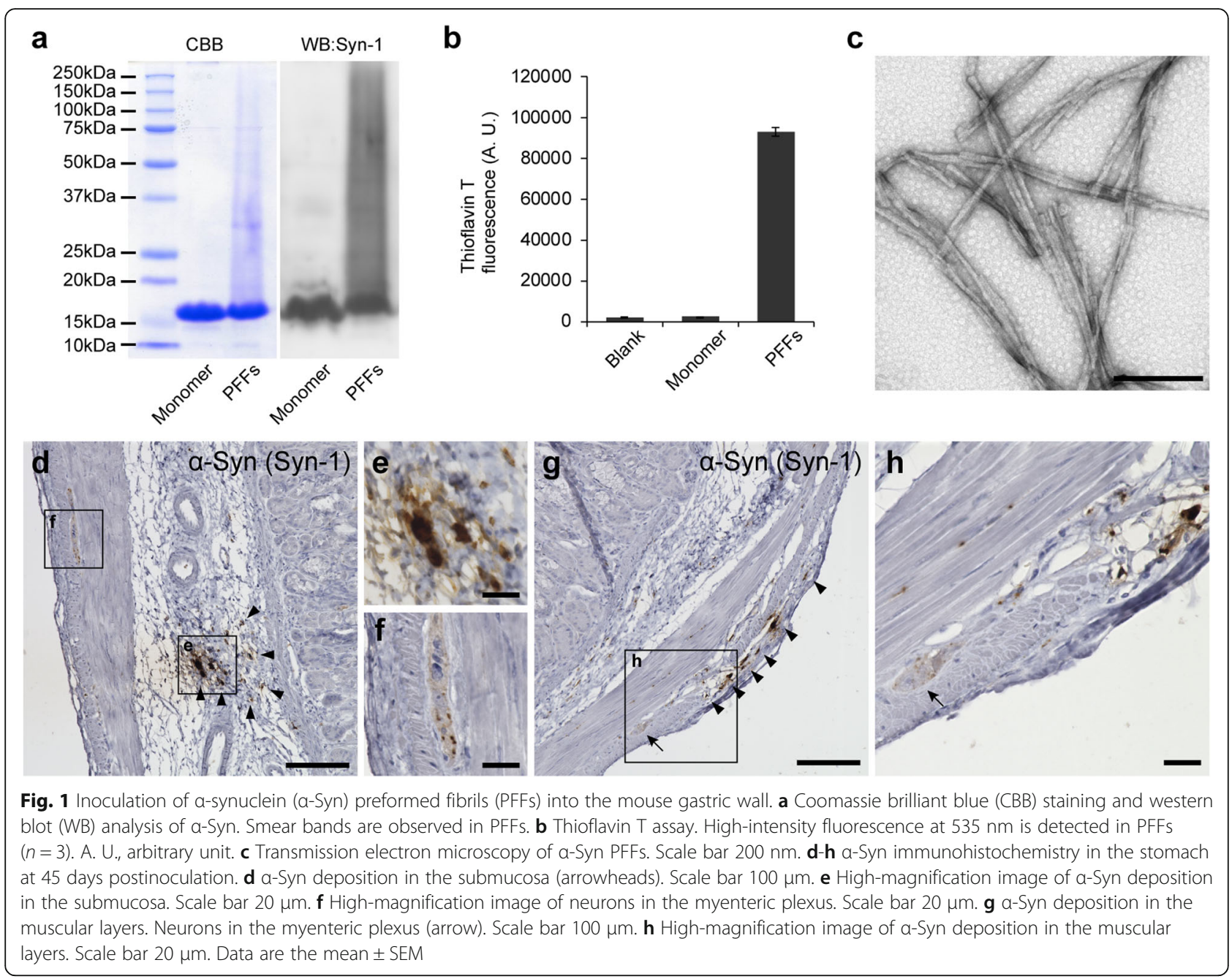

vagotomized mice 23 days after $\alpha$-Syn PFF inoculation in addition to 45 days. The numbers of ChAT-positive neurons on the vagotomy side decreased to $40 \%$ and to $20 \%$ compared with those of untreated mice 23 days and 45 days after surgery, respectively (Fig. $4 \mathrm{~b}, \mathrm{f}$ ). Although a certain number of ChAT-positive neurons survived on the vagotomy side, $\mathrm{p}-\alpha-$ Syn-positive neurons were observed only on the unvagotomized side at both 23 and 45 days (Fig. $4 \mathrm{~b}-\mathrm{e}, \mathrm{g}$ ). This suggests that the vagus nerve indeed was the pathway by which $\alpha$-Syn PFFs inoculated into the stomach resulted in formation of $\mathrm{p}-\alpha$-Syn aggregates in the dmX.

\section{Phosphorylated a-Syn-positive neurons decreased in number, and no further propagation was observed up to 12 months after $a-S y n$ PFFs inoculation}

Then, we analyzed p- $\alpha$-Syn pathology up to 12 months after $\alpha$-Syn PFF inoculation. Unexpectedly, the number of $\mathrm{p}-\alpha$-Syn-positive neurons in the $\mathrm{dmX}$ bilaterally decreased over time (Fig. 5a). No further propagation of p$\alpha$-Syn pathology beyond the $\mathrm{dmX}$ was observed during this period. At 12 months postinoculation, there were only a small number of $\mathrm{p}-\alpha$-Syn-positive aggregates in the $\mathrm{dmX}$, which appeared as denser inclusions than those observed at 45 days postinoculation (Fig. 5b). There were also a few $\mathrm{p}$ - $\alpha$-Syn-positive neurons in the myenteric plexus at 12 months postinoculation (Fig. 5c). We also examined the thoracic spinal cord but observed no $\mathrm{p}$ - $\alpha$-Syn pathology up to 12 months postinoculation (Additional file 3: Figure S3).

\section{No apparent cell-type specificity in neurons containing} p-a-Syn-positive aggregates in the myenteric plexus

An autopsy study of patients with PD reported that most LBs in the ENS are present in VIP-positive neurons [24]. According to Braak's hypothesis, Lewy pathology initially occurs in the ENS, suggesting that a specific type of neurons in the ENS may play a key role in the pathogenesis of PD. Finally, we therefore examined which types of myenteric neurons contained LB-like aggregates 12 months after $\alpha$-Syn PFF inoculation. Although single markers cannot completely distinguish the various types 


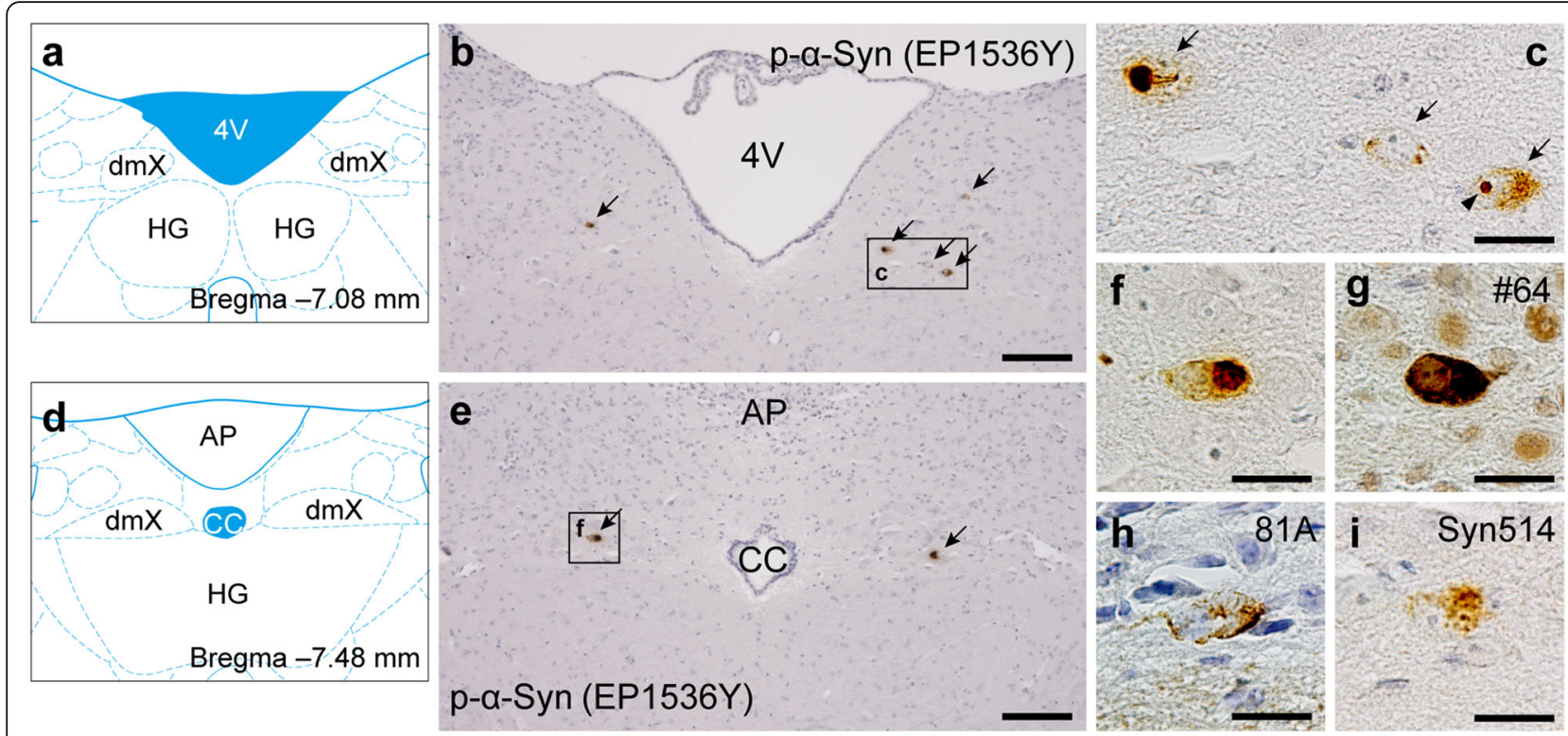

Fig. 2 Phosphorylated a-synuclein (p-a-Syn) pathology in the $d m X 45$ days after inoculation of a-Syn preformed fibrils. a Schematic of anatomy at bregma $-7.08 \mathrm{~mm}$ modified from [47]. dmX, dorsal motor nucleus of the vagus nerve; HG, hypoglossal nucleus; 4V, fourth ventricle. $\mathbf{b}$ P-a-Syn (EP1536Y) immunohistochemistry around bregma $-7.08 \mathrm{~mm}$. Scale bar $100 \mu \mathrm{m}$. c High-magnification image of p-a-Syn-positive cells (arrows). P-a-Syn-positive intranuclear dots are occasionally observed (arrowhead). Scale bar $20 \mu \mathrm{m}$. d Schematic of anatomy at bregma $-7.48 \mathrm{~mm}$. AP, area postrema; CC, central canal. e P-a-Syn (EP1536Y) immunohistochemistry around bregma $-7.48 \mathrm{~mm}$. Scale bar $100 \mu \mathrm{m}$. f High-magnification image of p-a-Syn-positive cells. Scale bar $20 \mu \mathrm{m}$. g-i Immunohistochemistry assessing p-a-Syn (\#64), p-a-Syn (81A), or nitrated a-Syn (Syn514) shows p-a-Syn-positive or nitrated a-Syn-positive cells in the dmX. Scale bar $20 \mu \mathrm{m}$

of neurons, many VIP-positive neurons in the myenteric plexus are inhibitory motor neurons and are also immunopositive for NOS1 (Fig. 6a, b) [25, 26]. Excitatory motor neurons are immunopositive for ChAT and substance P (Fig. 6c, d). We conducted double immunofluorescent analysis using antibodies against these markers along with anti-p- $\alpha$-Syn antibodies. A substantial portion of $\mathrm{p}$ - $\alpha$-Syn aggregates were present in VIPor NOS1-positive neurons (83\% [10/12] and 67\% [6/9], respectively; Fig. 6e-1), but some aggregates were also present in ChAT- or substance P-positive neurons (45\% [6/13] and 33\% [4/12], respectively; Fig. 6m-t). We therefore did not find cell-type specificity in neurons containing $\mathrm{p}$ - $\alpha$-Syn-positive aggregates in the myenteric plexus in this mouse model.

\section{Discussion}

A number of studies in both humans and animals have been conducted after Braak et al. published the "gut-tobrain" hypothesis, but controversy remains. In an wholebody autopsy study of several hundred cases, there was not a single case in which Lewy pathology was present in the ENS but not in the CNS, which argues against Braak's hypothesis [27]. On the other hand, two independent cohort studies reported that the risk of developing PD may be substantially decreased following truncal vagotomy, which does support the hypothesis by suggesting the involvement of the vagus nerve in the pathogenesis of PD $[28,29]$. In the present study, we investigated the progression of LB-like pathology as proposed by Braak's hypothesis by inoculating $\alpha$-Syn PFFs into the mouse gastric wall. Although the experimental conditions differ from the putative progression of Lewy pathology from the ENS to the $\mathrm{dmX}$ in human PD, we demonstrated that misfolded fibrillar forms of $\alpha$-Syn present in the gastric wall were capable of inducing LBlike pathology in the $\mathrm{dmX}$ via the vagus nerve. Unexpectedly, however, the number of neurons in the $\mathrm{dmX}$ containing $\mathrm{p}-\alpha$-Syn-positive aggregates decreased over time. It may be caused by the death of those neurons $[12,30]$ or chronological degradation of those aggregates.

According to the Braak's staging system, the neuroanatomical structures affected at stages 1,2, and 3 include the $\mathrm{dmX}$, locus coeruleus (LC), and SNpc, respectively. However, though brainstem nuclei are interconnected with each other directly or indirectly, it remains unclear what routes Lewy pathology follow from the $\mathrm{dmX}$, through the LC, to the SNpc in the human brainstem [15]. Identifying such routes is indispensable for a better understanding of the clinicopathologic progression of $\mathrm{PD}$, including the development of prodromal symptoms. Because the $\mathrm{dmX}$ receives input from a broad range of brain regions such as the solitary nucleus, LC, raphe nuclei, hypothalamus, and amygdala [31], we expected to 

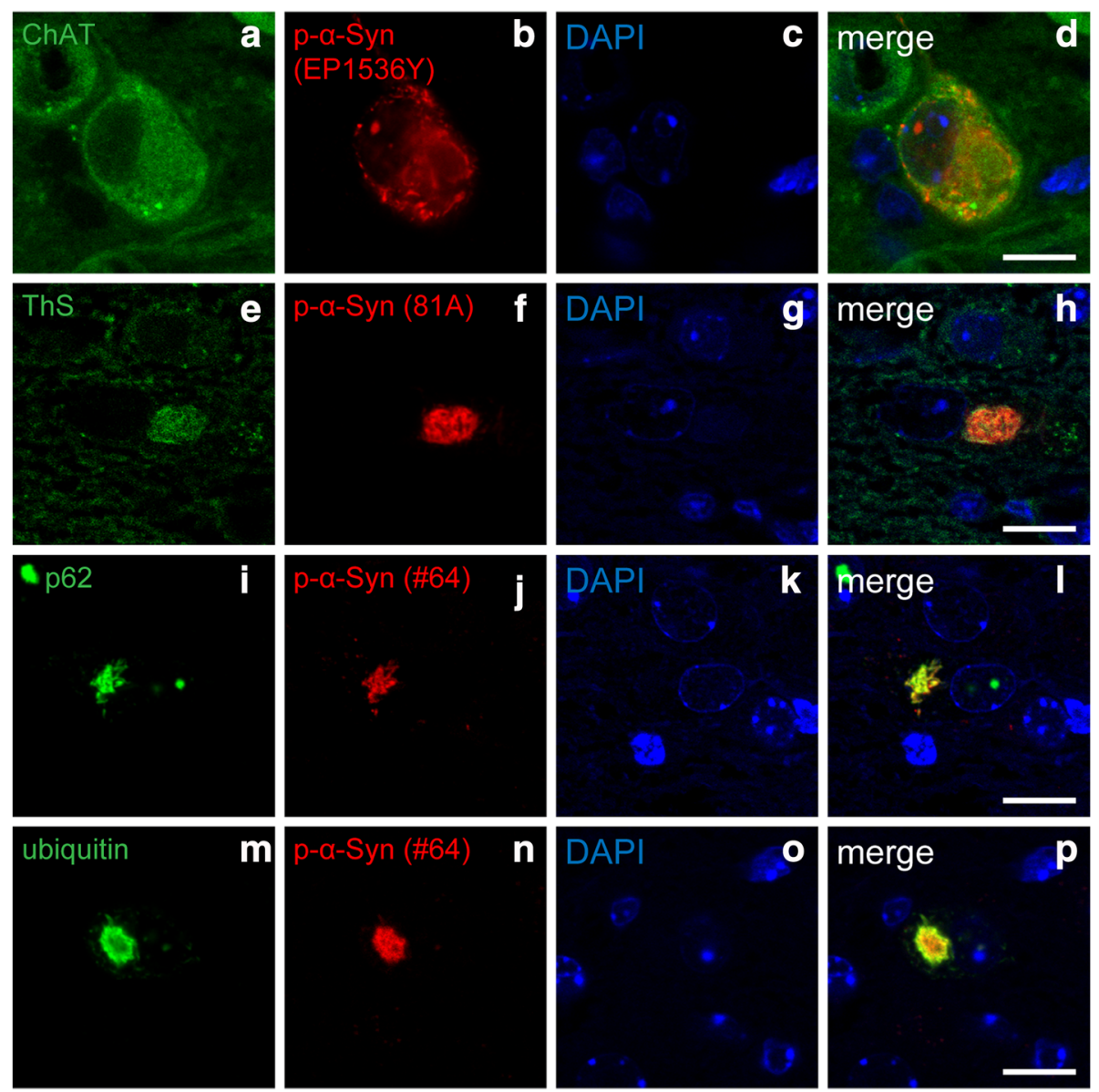

Fig. 3 Characterization of p-a-Syn pathology in the dmX 45 days after inoculation of a-Syn preformed fibrils. a-d Double immunostaining for choline acetyltransferase (ChAT) (green) and p-a-Syn (EP1536Y, red). Scale bar $10 \mu \mathrm{m}$. e-h Thioflavin S (ThS) staining (green) and immunostaining for p-a-Syn (81A, red). Scale bar $10 \mu \mathrm{m}$. i-I Double immunostaining for p62 (green) and p-a-Syn (\#64, red). Scale bar $10 \mu \mathrm{m}$. m-p Double immunostaining for ubiquitin (green) and p-a-Syn (\#64, red). Scale bar $10 \mu \mathrm{m}$

find $\mathrm{p}-\alpha$-Syn-positive aggregates in those structures as well. However, no $\mathrm{p}-\alpha$-Syn-positive aggregates were observed in any mouse brain region other than the $\mathrm{dmX}$ up to 12 months postinoculation. A recent study reported that $\alpha$-Syn PFF inoculation into the rat descending colon induced a small number of $\mathrm{p}-\alpha$-Syn-positive aggregates in the LC as well as $\operatorname{dmX}$ at 1 month postinoculation [32]. However, the study also reported that no p- $\alpha$-Syn-positive aggregates were found anywhere in the rat brain, including the $\mathrm{dmX}$ and LC, at later time points [32].

There are several possible interpretations of our observation that $\mathrm{p}-\alpha$-Syn pathology did not spread beyond the $\mathrm{dmX}$. First, some genetic or environmental factors may be required for further propagation, as sporadic PD is thought to be caused by the interaction of such factors. Indeed, it was shown that intracerebral inoculation of $\alpha$ Syn PFFs or tau fibrils induced spreading of $\alpha$-Syn or tau aggregates in interconnected brain regions of wild-type mice but, over time, the number of affected brain regions peaked $[33,34]$. These findings suggest that seeding pathological aggregates in wild-type mice is not sufficient to replicate natural disease progression, indicating that some additional factors are required for continuous spread of aggregates. For example, single nucleotide polymorphisms in SNCA (the $\alpha$-Syn gene) which increase $\alpha$-Syn expression have been identified as a genetic risk factor for PD [35]. Snca-null mice never show LB-like pathology or propagation of $\alpha$-Syn pathology when $\alpha$-Syn PFFs are inoculated into their brains [12], so it is conceivable that the opposite situation, that is, transgenic or virus-mediated overexpression of $\alpha-S y n$, might accelerate the formation and propagation of $\alpha$-Syn pathology. Mutations in GBA (the glucocerebrosidase gene), the strongest genetic risk factor for $\mathrm{PD}$, as well as aging, one of the environmental risk factors, both affect the proteolytic activity of the autophagy-lysosomal pathway, possibly promoting the formation and propagation of $\alpha$-Syn pathology [36, 37]. Both the environmental neurotoxin rotenone and the inflammogen lipopolysaccharide have been reported to induce intraneuronal $\alpha$ - 


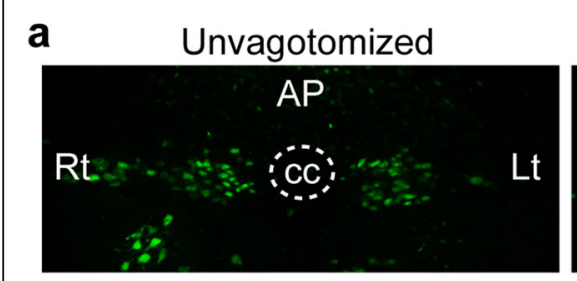

b Rt. vagotomized (23days)
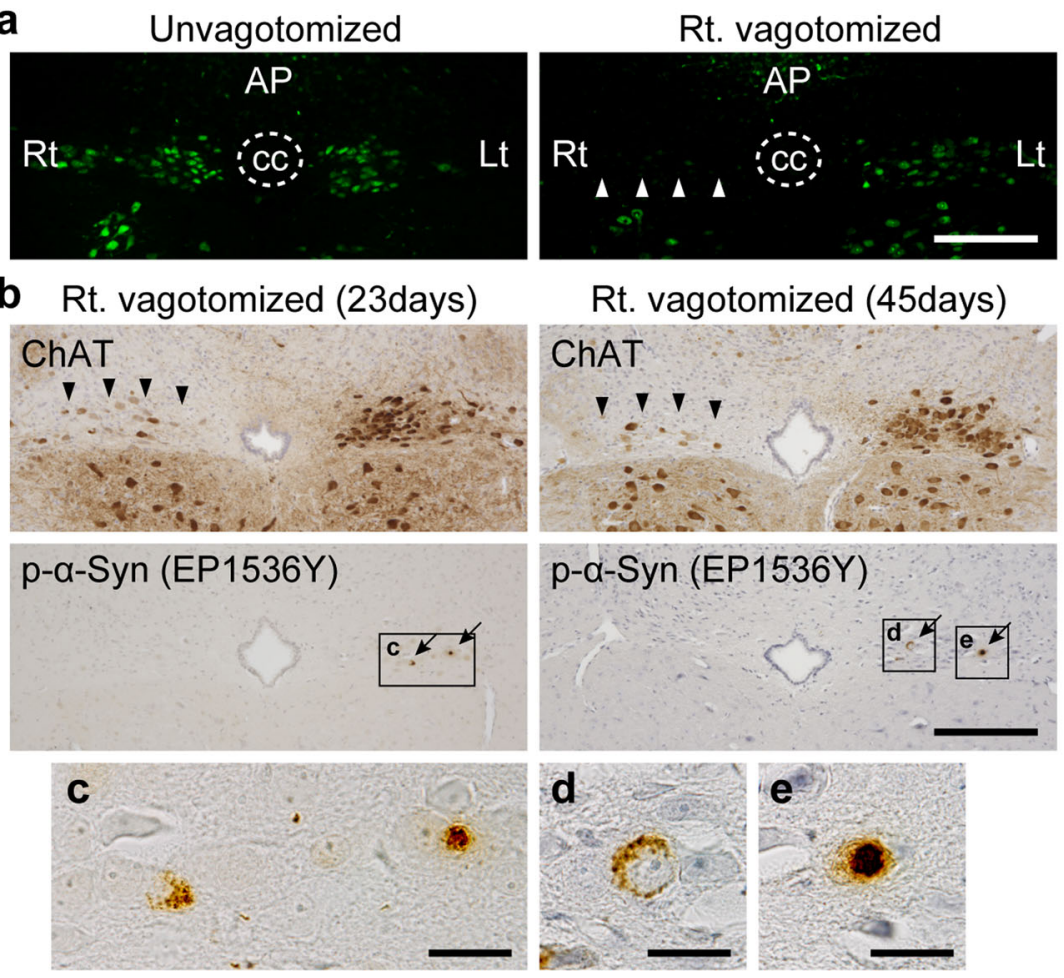

\section{Rt. vagotomized (45days)}
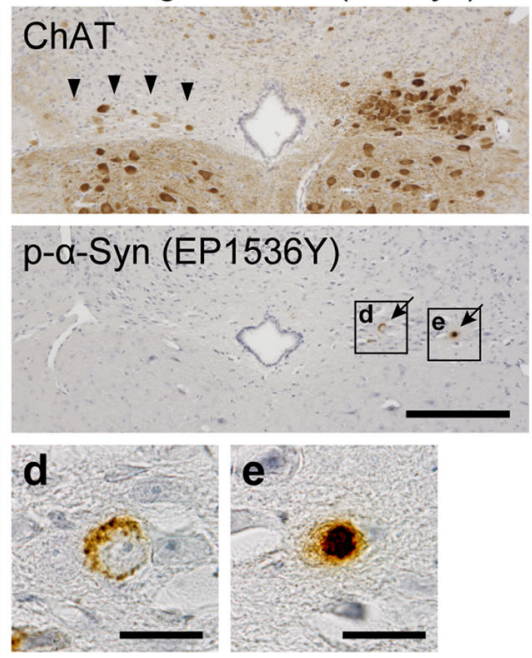

f

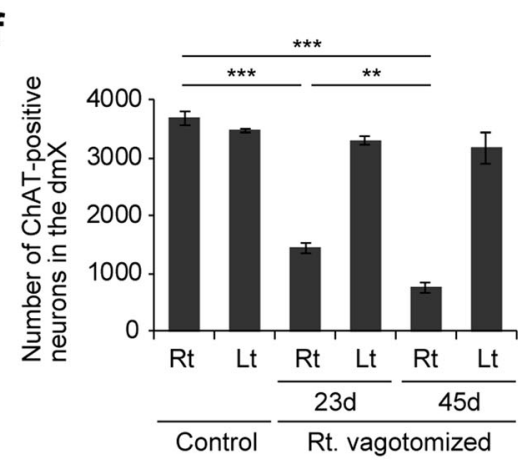

g

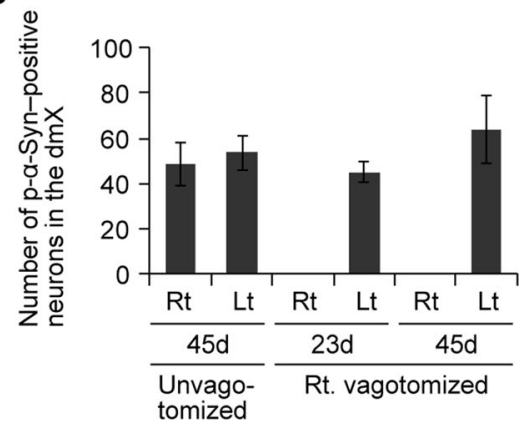

Fig. 4 Vagotomy prevents appearance of p-a-Syn pathology in the $d m X$ after inoculation of a-Syn preformed fibrils. a FluoroGold labeling of the $\mathrm{dmX}$. Left panel: Labeling appears in the $\mathrm{dmX}$ bilaterally 5 days after intraperitoneal injection of FluoroGold in unvagotomized mice. Right panel: FluoroGold labeling is absent in the right $\mathrm{dmX}$ when right (Rt.) vagotomy was performed prior to intraperitoneal injection of FluoroGold. AP, area postrema; cc, central canal. Scale bar $200 \mu \mathrm{m}$. b choline acetyltransferase (ChAT) and p-a-Syn immunohistochemistry in right-vagotomized mice 23 days and 45 days after a-Syn PFF inoculation. The number of ChAT-positive neurons in the right dmX decreased at 23 days and further decreased at 45 days (arrowheads). P-a-Syn-positive aggregates are present only in the left dmX at both time points (arrows). Scale bar $200 \mu m$. c-e High-magnification images of p-a-Syn-positive cells. Scale bars $20 \mu \mathrm{m}$. $\mathbf{f}$ Number of ChAT-positive neurons in the $\mathrm{dmX}$ of untreated mice (Control) and right-vagotomized mice 23 days and 45 days after a-Syn PFF inoculation $(n=3-4)$. One-way ANOVA with Tukey's multiplecomparisons test was performed; ${ }^{* *} p<0.01,{ }^{* * *} p<0.001$. g Number of $p$-a-Syn-positive neurons in the $\mathrm{dmX}$ of unvagotomised mice 45 days after a-Syn PFF inoculation and right-vagotomized mice 23 days and 45 days after a-Syn PFF inoculation $(n=4)$. Data are the mean \pm SEM
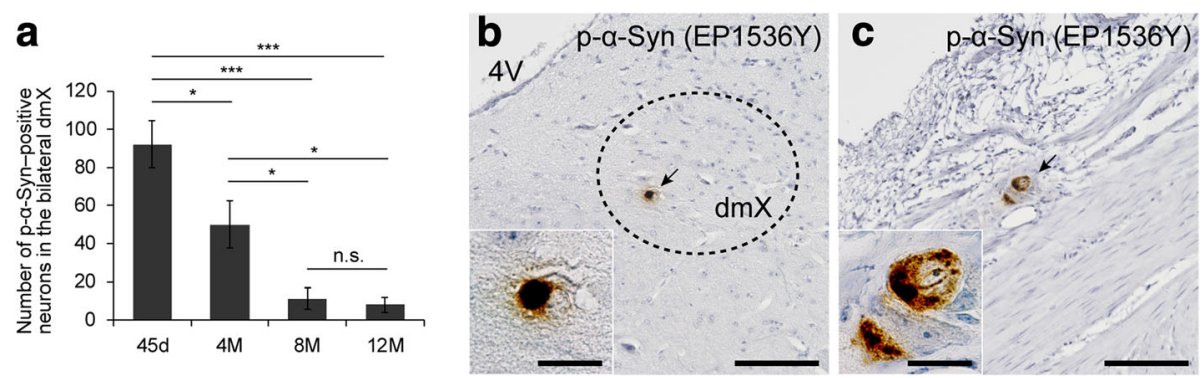

Fig. 5 Chronological analysis of p-a-Syn pathology after inoculation of a-Syn preformed fibrils. a Number of p-a-Syn-positive neurons in the dmX of mice bilaterally examined at 45 days, 4 months, 8 months, and 12 months postinoculation of a-Syn preformed fibrils $(n=5-8)$. One-way ANOVA with Tukey's multiple-comparisons test was performed; ${ }^{*} p<0.05,{ }^{* * *} p<0.001$, n.s. means not significant. b p-a-Syn (EP1536Y) immunohistochemistry in the dmX of a mouse at 12 months postinoculation. Dashed line outlines the approximate area of the $\mathrm{dmX}$. $\mathrm{dmX}$, dorsal motor nucleus of the vagus nerve; $4 \mathrm{~V}$, fourth ventricle. Scale bar $100 \mu \mathrm{m}$. A p-a-Syn-positive neuron (arrow) is enlarged in the inset. Scale bar $20 \mu \mathrm{m}$. c p-a-Syn (EP1536Y) immunohistochemistry in the myenteric plexus of a mouse at 12 months postinoculation. Scale bar $100 \mu \mathrm{m}$. A p-a-Syn-positive neuron (arrow) is enlarged in the inset. Scale bar $20 \mu \mathrm{m}$. Data are the mean \pm SEM 

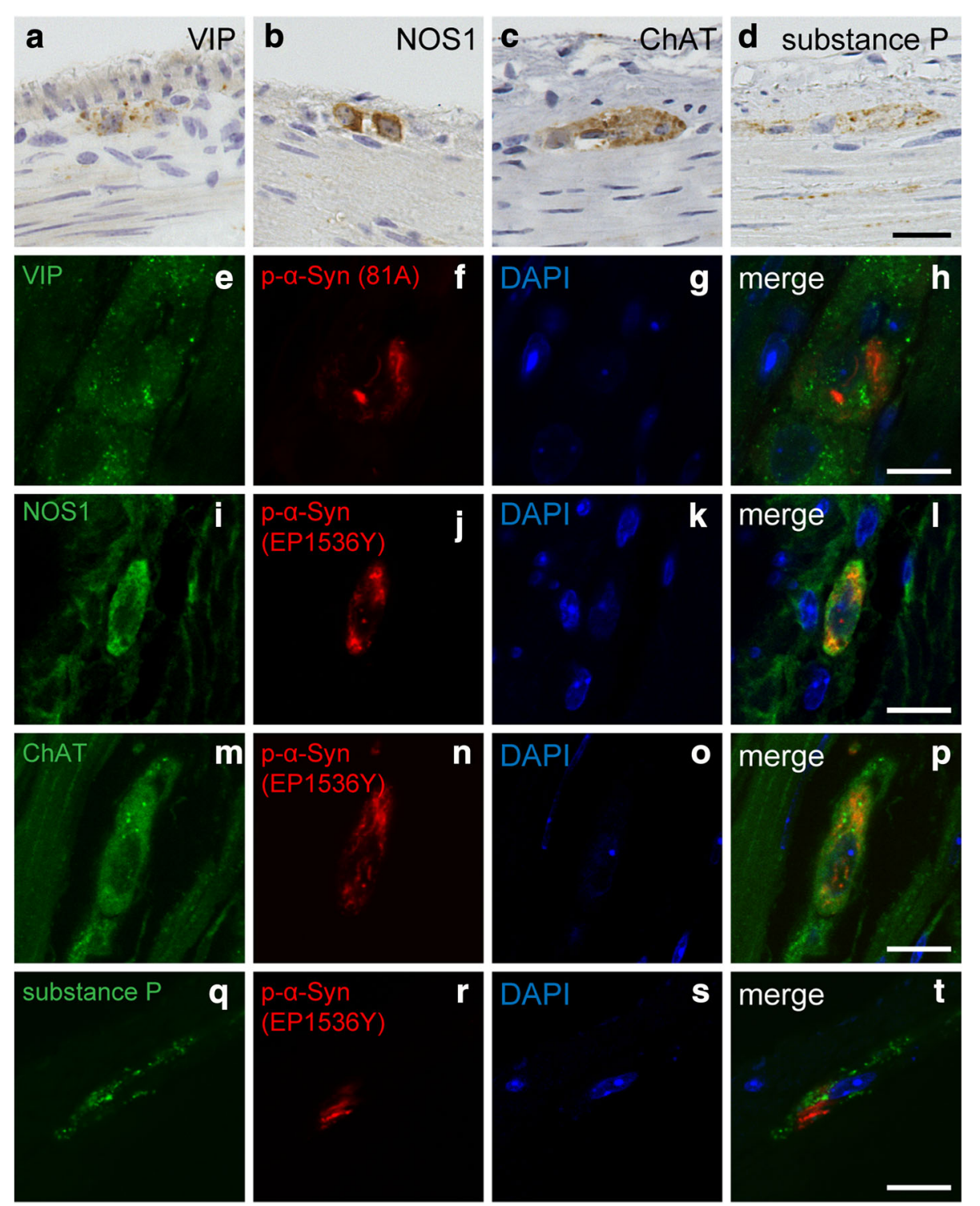

Fig. 6 Analysis of types of neurons containing p-a-Syn-positive aggregates in the myenteric plexus. a-d Representative images of vasoactive intestinal peptide (VIP), nitric oxide synthase 1 (NOS1), choline acetyltransferase (ChAT), and substance P immunohistochemistry in the myenteric plexus. e-h Double immunostaining for VIP (green) and p-a-Syn (81A, red). Scale bar 10 um. i-I Double immunostaining for NOS1 (green) and p-a-Syn (EP1536Y, red). Scale bar $10 \mu \mathrm{m}$. m-p Double immunostaining for ChAT (green) and p-a-Syn (EP1536Y, red). Scale bar $10 \mu \mathrm{m}$. q-t Double immunostaining for Substance P (green) and p-a-Syn (EP1536Y, red). Scale bar $10 \mu \mathrm{m}$

Syn accumulation in mouse brains, suggesting that they might also facilitate the propagation of $\alpha$-Syn pathology [38-40]. Therefore, including some of these factors along with $\alpha$-Syn PFF inoculation may enable us to replicate the progression of PD pathology in accordance with Braak's hypothesis.

Second, the $\alpha$-Syn PFFs used in this study may not have been potent enough to induce neuron-to-neuron propagation. Recent evidence has documented that recombinant $\alpha$-Syn monomers can form $\alpha$-Syn aggregates with a variety of conformations and biological activities [41]. Distinct $\alpha$-Syn aggregates named "strains" are associated with different pathologies and have variable seeding activity, cell toxicity, and cell-type preference both in vitro and in vivo [42, 43]. Importantly, distinct $\alpha$-Syn strains maintain their intrinsic conformations and properties even in seeded fibrillization reactions in vitro and in vivo $[43,44]$. These suggest that distinct $\alpha$-Syn strains probably have varying potency for inducing neuron-to-neuron propagation in the mouse brains. We occasionally observed uncommon pathologies in $\mathrm{PD}, \mathrm{p}-$ $\alpha$-Syn-positive dots in the neuronal nuclei in the $\mathrm{dmX}$ and $p-\alpha-S y n-$ positive aggregates in ChAT- or substance P-positive neurons in the ENS. These pathological 
features of this mouse model might be derived from different properties of $\alpha$-Syn PFFs from those of $\alpha$-Syn aggregates in PD. It may be worth trying to use other $\alpha$ Syn strains or $\alpha$-Syn aggregates purified from PD brains.

Finally, given that there are specific neural routes vulnerable to spread of $\alpha$-Syn aggregates, it may be that caudo-rostral routes originating from the $\mathrm{dmX}$ are less likely to be among those specific routes. Based on a CNS-wide survey of several hundred autopsy cases, Beach et al. proposed a unified staging system for LB diseases [45]. In this system, stage I is defined as involvement of only the olfactory bulb, whereas the brain stem is involved in later stages, II or III. It was reported that there are almost no cases of incidental LB pathology without olfactory bulb involvement as well as many that involve the olfactory bulb alone [27]. The authors hypothesized that $\alpha$-Syn pathology in peripheral organs emerges after that in the CNS because peripheral $\alpha$-Syn pathology was not observed in stage I [27]. A previous study demonstrated that virally overexpressed $\alpha$-Syn in the rat midbrain gained access into $\mathrm{dmX}$ neurons and then reached presynaptic terminals of the vagus nerve in the gastric wall, supporting this hypothesis [46]. Based on these observations and hypotheses, we may have to consider a rostro-caudal pathway as well as a caudorostral pathway of $\alpha$-Syn spread in future research.

\section{Conclusions}

In conclusion, this study demonstrated that inoculation of $\alpha$-Syn PFFs into the mouse gastric wall can induce LB-like pathology in the $\mathrm{dmX}$ via the vagus nerve, supporting Braak's "gut-to-brain" hypothesis. However, further caudo-rostral spread of $\alpha$-Syn pathology was not observed up to 12 months postinoculation. Other factors may be required for further progression of PD pathology beyond the $\mathrm{dmX}$. Our chronological, detailed analysis revealed similarities and dissimilarities of this mouse model to human PD and provides important clues for designing future research.

\section{Additional Files}

Additional file 1: Figure S1. Phosphorylated a-synuclein (p-a-Syn) pathology in the stomach 45 days after a-Syn preformed fibrils were inoculated into a mouse gastric wall. a P-a-Syn (EP1536Y) immunohistochemistry of the serial section from Fig. 1d. No apparent p-a-Syn pathology is seen. Scale bar $100 \mu \mathrm{m} . \mathbf{b}$ P-a-Syn (EP1536Y) immunohistochemistry of the serial section from Fig. 1g. No apparent $p$ - $a-S y n$ pathology is seen in the myenteric neurons. Scale bar $100 \mu \mathrm{m}$. c P-a-Syn (EP1536Y) immunohistochemistry of another section showing p-a-Syn aggregates in the myenteric plexus (arrow), enlarged in the inset. Scale bar $20 \mu \mathrm{m}$. (TIF 4963 kb)

Additional file 2: Figure S2. No phosphorylated a-synuclein (p-a-Syn) pathology is seen in either mouse brain or stomach 45 days after phosphate-buffered saline inoculation into a mouse gastric wall. a P-a-Syn (EP1536Y) immunohistochemistry of a section around bregma $-7.08 \mathrm{~mm}$. Scale bar $100 \mu \mathrm{m}$. 4V, fourth ventricle. b P-a-Syn (EP1536Y) immunohistochemistry of a section around bregma - $7.48 \mathrm{~mm}$. Scale bar $100 \mu \mathrm{m}$. AP, area postrema; cc, central canal. c P-aSyn (EP1536Y) immunohistochemistry in the stomach. Scale bar $100 \mu \mathrm{m}$. (TIF 7863 kb)

Additional file 3: Figure S3. No phosphorylated a-synuclein ( $p-a-S y n)$ pathology in the thoracic spinal cord 12 months after inoculation of a-Syn preformed fibrils into the mouse gastric wall. a P-a-Syn (EP1536Y) immunohistochemistry of the thoracic spinal cord. Scale bar $100 \mu \mathrm{m}$. (TIF 3586 kb)

\section{Abbreviations}

4V: fourth ventricle; a-Syn: a-synuclein; A. U.: arbitrary unit; AP: Area postrema; CBB: Coomassie Brilliant Blue; cc: central canal; ChAT: Choline acetyltransferase; CNS: Central nervous system; dmX: dorsal motor nucleus of the vagus nerve; ENS: Enteric nervous system; HG: Hypoglossal nucleus; LB: Lewy body; NOS1: Nitric oxide synthase 1; PAGE: Polyacrylamide gel electrophoresis; PBS: Phosphate buffer saline; PBS: Phosphate-buffered saline; PD: Parkinson's disease; PFA: Paraformaldehyde; PFF: Preformed fibril; p-a-Syn: phosphorylated a-synuclein; SDS: Sodium dodecyl sulfate; ThS: Thioflavin S; VIP: Vasoactive intestinal polypeptide; WB: western blot

\section{Acknowledgements}

The authors are grateful to Dr. Masato Hasegawa (Tokyo Metropolitan Institute of Medical Science, Tokyo, Japan) for providing the plasmid pRK172 encoding mouse a-Syn cDNA sequence and to Ms. Rie Hikawa (Kyoto University) for the excellent technical assistance.

\section{Funding}

This research is supported by the program for Brain Mapping by Integrated Neurotechnologies for Disease Studies (Brain/MINDS) from Ministry of Education, Culture, Sports Science, MEXT, and the Japan Agency for Medical Research and Development, AMED under the Grant Number JP17dm0207020h0004 (RT), JSPS KAKENHI Grant Numbers JP17H05698 (RT)، JP17K16119 (NU), the Takeda Science Foundation (NU), and the Kanae Foundation for the Promotion of Medical Science (NU).

Availability of data and materials

The manuscript has data included as electronic Additional files.

\section{Authors' contributions}

$\mathrm{NU}$ and RT conceived and designed the experiments. NU performed the experiments and analyzed the data. HiY performed the TEM experiments. NU and RT wrote the manuscript after fruitful discussion with MTU, YH, and HoY. All authors read and approved the final manuscript.

\section{Ethics approval}

The Animal Research Committee of Kyoto University granted ethical approval and permission (MedKyo 17,184).

\section{Competing interests}

The authors declare that they have no competing interests.

\section{Publisher's Note}

Springer Nature remains neutral with regard to jurisdictional claims in published maps and institutional affiliations.

\section{Author details}

${ }^{1}$ Department of Neurology, Kyoto University Graduate School of Medicine, 54 Shogoin-Kawaharacho, Kyoto, Sakyoku 606-8507, Japan. ${ }^{2}$ Center for Research on Green Sustainable Chemistry, Tottori University, 4-101

Koyamacho-minami, Tottori, Tottori 680-8550, Japan.

Received: 9 March 2018 Accepted: 2 May 2018

Published online: 11 May 2018

References

1. de Lau LML, Breteler MMB. Epidemiology of Parkinson's disease. Lancet Neurol. 2006;5:525-35. 
2. Dickson DW, Braak H, Duda JE, Duyckaerts C, Gasser T, Halliday GM, Hardy J, Leverenz JB, Del Tredici K, Wszolek ZK, Litvan I. Neuropathological assessment of Parkinson's disease: refining the diagnostic criteria. Lancet Neurol. 2009:8:1150-7.

3. Kalia LV, Lang AE. Parkinson's disease. Lancet. 2015;386:896-912.

4. Berg D, Postuma RB, Adler CH, Bloem BR, Chan P, Dubois B, Gasser T, Goetz CG, Halliday G, Joseph L, et al. MDS research criteria for prodromal Parkinson's disease. Mov Disord. 2015;30:1600-11.

5. Postuma RB, Aarsland D, Barone P, Burn DJ, Hawkes CH, Oertel W, Ziemssen T. Identifying prodromal Parkinson's disease: pre-motor disorders in Parkinson's disease. Mov Disord. 2012;27:617-26.

6. Braak H, Del Tredici K, Rub U, de Vos RA, Jansen Steur EN, Braak E. Staging of brain pathology related to sporadic Parkinson's disease. Neurobiol Aging. 2003;24:197-211.

7. Del Tredici K, Rub U, De Vos RA, Bohl JR, Braak H. Where does parkinson disease pathology begin in the brain? J Neuropathol Exp Neurol. 2002;61:413-26.

8. Braak H, de Vos RA, Bohl J, Del Tredici K. Gastric alpha-synuclein immunoreactive inclusions in Meissner's and Auerbach's plexuses in cases staged for Parkinson's disease-related brain pathology. Neurosci Lett. 2006; 396:67-72.

9. Hawkes $\mathrm{CH}$, Del Tredici K, Braak H. Parkinson's disease: a dual-hit hypothesis. Neuropathol Appl Neurobiol. 2007;33:599-614.

10. Brundin P, Li JY, Holton JL, Lindvall O, Revesz T. Research in motion: the enigma of Parkinson's disease pathology spread. Nat Rev Neurosci. 2008;9: $741-5$.

11. Angot E, Steiner JA, Hansen C, Li JY, Brundin P. Are synucleinopathies prionlike disorders? Lancet Neurol. 2010;9:1128-38.

12. Luk KC, Kehm V, Carroll J, Zhang B, O'Brien P, Trojanowski JQ, Lee VM. Pathological alpha-synuclein transmission initiates Parkinson-like neurodegeneration in nontransgenic mice. Science. 2012;338:949-53.

13. Masuda-Suzukake M, Nonaka T, Hosokawa M, Oikawa T, Arai T, Akiyama H, Mann DM, Hasegawa M. Prion-like spreading of pathological alphasynuclein in brain. Brain. 2013;136:1128-38.

14. Lionnet A, Leclair-Visonneau L, Neunlist M, Murayama S, Takao M, Adler CH, Derkinderen P, Beach TG. Does Parkinson's disease start in the gut? Acta Neuropathol. 2018;135:1-12.

15. Uchihara T, Giasson BI. Propagation of alpha-synuclein pathology: hypotheses, discoveries, and yet unresolved questions from experimental and human brain studies. Acta Neuropathol. 2016;131:49-73.

16. Holmqvist S, Chutna O, Bousset L, Aldrin-Kirk P, Li W, Bjorklund T, Wang ZY, Roybon L, Melki R, Li JY. Direct evidence of Parkinson pathology spread from the gastrointestinal tract to the brain in rats. Acta Neuropathol. 2014; 128:805-20.

17. Ulusoy A, Rusconi R, Perez-Revuelta BI, Musgrove RE, Helwig M, WinzenReichert B, Di Monte DA. Caudo-rostral brain spreading of alpha-synuclein through vagal connections. EMBO Mol Med. 2013;5:1119-27.

18. Masuda-Suzukake M, Nonaka T, Hosokawa M, Kubo M, Shimozawa A, Akiyama H, Hasegawa M. Pathological alpha-synuclein propagates through neural networks. Acta Neuropathol Commun. 2014;2:88.

19. Lee BR, Kamitani T. Improved immunodetection of endogenous alphasynuclein. PLoS One. 2011;6:e23939.

20. Phillips RJ, Walter GC, Wilder SL, Baronowsky EA, Powley TL. Alphasynuclein-immunopositive myenteric neurons and vagal preganglionic terminals: autonomic pathway implicated in Parkinson's disease? Neuroscience. 2008;153:733-50

21. Rey NL, Steiner JA, Maroof N, Luk KC, Madaj Z, Trojanowski JQ, Lee VM, Brundin P. Widespread transneuronal propagation of alpha-synucleinopathy triggered in olfactory bulb mimics prodromal Parkinson's disease. J Exp Med. 2016;213:1759-78.

22. Duda JE, Giasson BI, Mabon ME, Lee VM, Trojanowski JQ. Novel antibodies to synuclein show abundant striatal pathology in Lewy body diseases. Ann Neurol. 2002;52:205-10.

23. Yamada J, Hayashi Y, Jinno S, Wu Z, Inoue K, Kohsaka S, Nakanishi H. Reduced synaptic activity precedes synaptic stripping in vagal motoneurons after axotomy. Glia. 2008;56:1448-62.

24. Wakabayashi K, Takahashi H, Ohama E, Ikuta F. Parkinson's disease: an immunohistochemical study of Lewy body-containing neurons in the enteric nervous system. Acta Neuropathol. 1990;79:581-3.

25. Brookes SJ. Classes of enteric nerve cells in the Guinea-pig small intestine. Anat Rec. 2001:262:58-70.

26. Furness JB: The Enteric Nervous System. 1st edn: Wiley-Blackwell; 2006.
27. Adler $\mathrm{CH}$, Beach TG. Neuropathological basis of nonmotor manifestations of Parkinson's disease. Mov Disord. 2016:31:1114-9.

28. Svensson E, Horvath-Puho E, Thomsen RW, Djurhuus JC, Pedersen L, Borghammer P, Sorensen HT. Vagotomy and subsequent risk of Parkinson's disease. Ann Neurol. 2015;78:522-9.

29. Liu B, Fang F, Pedersen NL, Tillander A, Ludvigsson JF, Ekbom A, Svenningsson $P$, Chen H, Wirdefeldt K. Vagotomy and Parkinson disease: a Swedish registerbased matched-cohort study. Neurology. 2017;88:1996-2002.

30. Osterberg VR, Spinelli KJ, Weston LJ, Luk KC, Woltjer RL, Unni VK. Progressive aggregation of alpha-synuclein and selective degeneration of lewy inclusion-bearing neurons in a mouse model of parkinsonism. Cell Rep. 2015;10:1252-60.

31. Borghammer P. How does parkinson's disease begin? Perspectives on neuroanatomical pathways, prions, and histology. Mov Disord. 2018;33:48-57.

32. Manfredsson FP, Luk KC, Benskey MJ, Guezer A, Garcia J, Kuhn NC, Sandoval IM, Patterson JR, O'Mara a, Yonkers R, Kordower JH: Induction of alphasynuclein pathology in the enteric nervous system of the rat and nonhuman primate results in gastrointestinal dysmotility and transient CNS pathology. Neurobiology of disease 2018.

33. Rey NL, George S, Steiner JA, Madaj Z, Luk KC, Trojanowski JQ, Lee VM, Brundin P. Spread of aggregates after olfactory bulb injection of alphasynuclein fibrils is associated with early neuronal loss and is reduced long term. Acta Neuropathol. 2018;135:65-83.

34. Guo JL, Narasimhan S, Changolkar L, He Z, Stieber A, Zhang B, Gathagan RJ, Iba M, McBride JD, Trojanowski JQ, Lee VM. Unique pathological tau conformers from Alzheimer's brains transmit tau pathology in nontransgenic mice. J Exp Med. 2016;213:2635-54.

35. Soldner F, Stelzer Y, Shivalila CS, Abraham BJ, Latourelle JC, Barrasa MI, Goldmann J, Myers RH, Young RA, Jaenisch R. Parkinson-associated risk variant in distal enhancer of alpha-synuclein modulates target gene expression. Nature. 2016;533:95-9.

36. Collier TJ, Kanaan NM, Kordower JH. Ageing as a primary risk factor for Parkinson's disease: evidence from studies of non-human primates. Nat Rev Neurosci. 2011;12:359-66.

37. Mazzulli JR, Xu YH, Sun Y, Knight AL, McLean PJ, Caldwell GA, Sidransky E, Grabowski GA, Krainc D. Gaucher disease glucocerebrosidase and alphasynuclein form a bidirectional pathogenic loop in synucleinopathies. Cell. 2011;146:37-52.

38. Pan-Montojo F, Schwarz M, Winkler C, Arnhold M, O'Sullivan GA, Pal A, Said J, Marsico G, Verbavatz JM, Rodrigo-Angulo M, et al. Environmental toxins trigger PD-like progression via increased alpha-synuclein release from enteric neurons in mice. Sci Rep. 2012;2:898.

39. Gao HM, Zhang F, Zhou H, Kam W, Wilson B, Hong JS. Neuroinflammation and alpha-synuclein dysfunction potentiate each other, driving chronic progression of neurodegeneration in a mouse model of Parkinson's disease. Environ Health Perspect. 2011;119:807-14.

40. Lema Tome CM, Tyson T, Rey NL, Grathwohl S, Britschgi M, Brundin P. Inflammation and alpha-synuclein's prion-like behavior in Parkinson's disease-is there a link? Mol Neurobiol. 2013;47:561-74.

41. Peng C, Gathagan RJ, Lee VM. Distinct alpha-Synuclein strains and implications for heterogeneity among alpha-Synucleinopathies. Neurobiol Dis. 2018;109:209-18.

42. Guo JL, Covell DJ, Daniels JP, Iba M, Stieber A, Zhang B, Riddle DM, Kwong LK, Xu Y, Trojanowski JQ, Lee VM. Distinct alpha-synuclein strains differentially promote tau inclusions in neurons. Cell. 2013;154:103-17.

43. Peelaerts W, Bousset L, Van der Perren A, Moskalyuk A, Pulizzi R, Giugliano M, Van den Haute C, Melki R. Baekelandt V: alpha-Synuclein strains cause distinct synucleinopathies after local and systemic administration. Nature. 2015;522:340-4.

44. Luk KC, Covell DJ, Kehm VM, Zhang B, Song IY, Byrne MD, Pitkin RM, Decker SC, Trojanowski JQ, Lee VM. Molecular and biological compatibility with host alpha-Synuclein influences fibril pathogenicity. Cell Rep. 2016;16:3373-87.

45. Beach TG, Adler CH, Lue L, Sue LI, Bachalakuri J, Henry-Watson J, Sasse J, Boyer S, Shirohi S, Brooks R, et al. Unified staging system for Lewy body disorders: correlation with nigrostriatal degeneration, cognitive impairment and motor dysfunction. Acta Neuropathol. 2009;117:613-34.

46. Ulusoy A, Phillips RJ, Helwig M, Klinkenberg M, Powley TL, Di Monte DA. Brain-to-stomach transfer of alpha-synuclein via vagal preganglionic projections. Acta Neuropathol. 2017;133:381-93.

47. Franklin K, Paxinos G: The Mouse Brain in Stereotaxic Coordinates. Compact 3rd Edition edn: Academic Press; 2008. 